MacCoun, R. \& Reuter, P. (200I) Evaluating alternative cannabis regimes. British Journal of Psychiatry, 178, $123-128$

de Zwart, W. M., Monshouwer, K. \& Smit, F. (2000) Cannabis use among Dutch students has stabilised. http: //www.trimbos.nl

W. de Zwart, M. van Laar Trimbos-institute, Netherlands Institute of Mental Health and Addiction, PO 725, 3500 AS Utrecht, The Netherlands

\section{Effects of schizophrenia on patients' relatives}

Tennakoon et al (2000) stated that their study is 'one of the first' to investigate burden among caregivers of people with first-episode psychosis. Their paper was published 13 years after our paper which covered much the same ground (The Scottish Schizophrenia Research Group, 1987) and which they did not mention.

We found, using the General Health Questionnaire (GHQ), that $24(77 \%)$ of 31 main caregivers were categorised as 'psychiatric cases'; this compares with $12 \%$ of caregivers in the Tennakoon et al study, which also used the GHQ. However, the relatives in our study were interviewed during the first week of the patients' first admission to hospital, and before the patients received antipsychotic medication. Tennakoon et al's patients could have been ill for up to 2 years and received up to 12 weeks of antipsychotic drugs - hardly 'first-episode'.

In our 5-year follow-up study (The Scottish Schizophrenia Research Group, 1992), 14 of the 19 relatives who were still living with the patient were reassessed; 6 $(43 \%)$ were still categorised as 'cases', using the GHQ. We concluded that a patient's illness had a considerable and continuing effect on his or her relatives.

The Scottish Schizophrenia Research Group (1987) The Scottish First Episode Schizophrenia Study IV.

Psychiatric and social impact on relatives. British Journal of Psychiatry, 150, 340-344.

- (1992) The Scottish First Episode Schizophrenia Study VIII. Five-year follow-up: clinical and psychosocial findings. British Journal of Psychiatry, 16I, 496-500.

Tennakoon, L., Fannon, D., Doku, V., et al (2000) Experience of caregiving: relatives of people experiencing a first episode of psychosis. British Journal of Psychiatry, 177, 529-533.

R. G. McCreadie Department of Clinical Research, Crichton Royal Hospital, Dumfries DGI 4TG

\section{Over-representation of Black people in secure psychiatric facilities}

I read with great interest Lelliot et al's (2001) survey of patients from an innerLondon health authority in medium secure psychiatric care. In particular, the authors set out to compare Black and White patients and found statistical differences which they dismiss. The scores on the Health of the Nation Outcome Scales and compound variables of clinical behaviour and social function differed between Black and White patients, but Lelliot et al comment that these may not be clinically significant.

If statistically significant findings on scales used in the study need not be explained, then the instruments cannot be considered valid to address the third aim of the study, to compare Black and White patients. Black patients were significantly less morbid on a number of clinical, social and behavioural variables, including affective symptoms, activities of daily living, problems with living skills, relationship problems and other mental or behavioural problems of self-harm and overactive and aggressive behaviour. Why should Black patients with less severe psychopathology or aggressive behaviour continue to find themselves in medium secure units?

There are two possible explanations. One is non-engagement with treatment options in less secure environments. It is known that Black patients are more likely to abscond from in-patient units (Falkowski et al, 1990) and that they are increasingly dissatisfied with each consecutive in-patient admission (Parkman et al, 1997).

An alternative explanation is that Black patients are perceived to be more dangerous despite lower ratings of psychopathology (Cope, 1990; Lewis et al, 1990). Lelliot et al unfortunately dismiss important findings as clinically insignificant. These very findings warrant further research and exploration and such work may well deliver a better understanding of why Black people are over-represented in secure psychiatric facilities.

Cope, R. (1990) Psychiatry, ethnicity \& crime. In Forensic Psychiatry (eds R. Bluglass \& P. Bowden). London: Churchill Livingstone.

Falkowski, J., Watts, V., Falkowski, W., et al (1990) Patients leaving hospital without the knowledge or permission of staff - absconding. British Journal of Psychiatry, 156, 488-490.

Lelliott, P., Audini, B. \& Duffett, R. (200I) Survey of patients from an inner-London health authority in medium secure psychiatric care. British Journal of Psychiatry, 178, 62-66.

Lewis, G., Croft-Jeffreys, C. \& David, A. (1990) Are British psychiatrists racist? British Journal of Psychiatry, I57, 4I0-4I5.

Parkman, S., Davies, S., Leese, M., et al (1997) Ethnic differences in satisfaction with mental health services among representative people with psychosis in South London: PRiSM Study 4. British Journal of Psychiatry, I7I, 260-264.

K. Bhui Department of Psychiatry,

St Bartholomew's and The Royal London School of Medicine and Dentistry, Queen Mary \& Westfield College, University of London, Mile End Road, London El 4NS

\section{Evidence-based psychiatry}

Lawrie et al (2001) have touched upon the core problem of evidence-based psychiatry by raising the most relevant questions. Although the evidence-based medicine movement began in 1992 (Sackett et al, 2000), it was not until the Royal College of Psychiatrists introduced a Critical Review Paper to the MRCPsych Part II examination in 1999 that clinicians suddenly realised the problems of not knowing enough about critical appraisal of scientific papers.

Brown \& Wilkinson (2000) assert, "Psychiatrists should be able to evaluate published literature both in terms of its scientific validity and its clinical relevance". Why - to be able to practise evidence-based psychiatry, or to help trainees pass their exam? In a Scottish survey Lawrie et al (2000) discovered that senior psychiatrists found the time required to search and appraise the literature as the greatest barrier to practising evidence-based psychiatry. Would they be able to practise better if they had enough time, for instance 60 minutes per day? I do not know how to search for the best evidence to answer this question. I have recently read the recommended books (Brown \& Wilkinson, 2000; Sackett et al, 2000), and I have also attended a few evidence-based medicine workshops. When my patients and trainees ask reallife clinical questions, I often get lost. Is this the beginning of my ageing-related cognitive impairment? Or is it just because I am such a busy clinician that I do not have time and need to take evidence-based psychiatry seriously? But how can we help our trainees? What about our own revalidation? We cannot really fudge the issue any more if we want to remain effective trainers. 